$$
f+\lambda g=\phi(\lambda), g=1+\sum_{i=2}^{p} \frac{b_{i}{ }^{2}}{\left(\lambda-\lambda_{i}\right)^{2}}-\sum_{j=p+1}^{n} \frac{b_{j}^{2}}{\left(\lambda_{j}-\lambda\right)^{2}}=\phi^{\prime}(\lambda),
$$

a rational function of $\lambda$ continuous in the interval (11).

If all the $b_{k} \neq 0$ we have $\phi^{\prime}\left(\lambda_{p+1}\right)=-\infty, \phi^{\prime}\left(\lambda_{p}\right)=\infty$ if $p>1$, while if $p=1$, then $\phi^{\prime}(-\infty)=1>0$. Hence there exists a $\lambda$ in the intervals (10) such that $\phi^{\prime}(\lambda)=g=0$. But then our hypothesis states that $f=\phi(\lambda)>0$. By (12), and since $\phi(\lambda)>0$, we have $f+\lambda g$ positive definite.

There remains the case where some $b_{k}=0$. Here we may permute the $x_{i}$ and change the sign of $g$ if necessary and carry the corresponding $x_{k}$ into $x_{1}$. Then $f=-\lambda_{1} x_{1}^{2}+f_{0}\left(x_{2}, \cdots, x_{n}\right)$. As in the proof above we may carry $f_{0}$ into (7) and have $f$ in the form (3). But $f>0$ for $g=0$ and as in the proof of Lemma 2 we have (5), and $f+\lambda g$ is positive definite for $\lambda$ as in (6).

We have proved our theorem. Notice that our reduction to the case $g$ non-singular together with Lemmas 1,2 determines the range of $\lambda$ for which $f+\lambda g$ is positive definite.

The University of Chicago

\title{
THE RIEMANNIAN CURVATURE OF A HYPERSURFACE*
}

\section{AARON FIALKOW $\dagger$}

1. Introduction. It is a well known theorem of Gauss that the total curvature of any two dimensional surface in euclidean three space is equal to the product of the principal normal curvatures. Eisenhart $\ddagger$ has shown that a generalization of this theorem applies to Riemann spaces of class one; that is, the hypersurfaces of an $n$-dimensional flat space. He proves the theorem:

When the lines of curvature of a Riemann space $V_{n}$ of class one are real and none of them is tangent to a null vector, the Riemannian curvature at a point for the orientation determined by the direction of two lines of curvature at the point is numerically equal to the product of the corresponding normal curvatures; the sign is determined by the character of the normal to $V_{n}$ in the enveloping flat $V_{n+1}$.

* Presented to the Society, September 10, 1937.

$\dagger$ National Research Fellow.

$\ddagger$ L. P. Eisenhart, Riemannian Geometry, 1926, p. 199. 
This is Gauss' theorem for a flat $V_{n+1}$. The analogous theorem, which is true of the hypersurfaces of any Riemann space, appears to have been overlooked and is derived in $\$ 3$ of this note. From this result, we establish the following theorem which is the converse of the theorem of Gauss for a flat $V_{n+1}$ :

Let $V_{n}$ be any hypersurface of a $V_{n+1}$ such that the lines of curvature of $V_{n}$ are real and none of them is tangent to a null vector. Let the Riemannian curvature of $V_{n}$ at a point for the orientation determined by the direction of two lines of curvature at the point be numerically equal to the corresponding normal curvatures, the sign being determined by the character of the normal to $V_{n}$ in the enveloping $V_{n+1}$. Then $V_{n+1}$ is a flat space.

2. Hypersurfaces of a $V_{n+1}$. We begin by a short summary of those portions of the theory of hypersurfaces which are necessary in our work. Let $V_{n+1}$ be a real Riemann space with the first fundamental form*

$$
d s^{2}=a_{\alpha \beta} d y^{\alpha} d y^{\beta}
$$

Any real hypersurface $V_{n}$ immersed in $V_{n+1}$ is defined by a system of equations $y^{\alpha}=y^{\alpha}\left(x^{1}, x^{2}, \cdots, x^{n}\right)$, where the rank of the Jacobian matrix $\left\|\partial y^{\alpha} / \partial x^{i}\right\|$ is $n$. The metric induced in the hypersurface by (1) determines the first fundamental form of $V_{n}$ as

$$
d s^{2}=g_{i j} d x^{i} d x^{j}
$$

where

$$
g_{i j}=a_{\alpha \beta} y_{, i}^{\alpha} y_{, j}^{\beta} \cdot \dagger
$$

We assume that real coordinate systems $\left\{y^{\alpha}\right\}$ and $\left\{x^{i}\right\}$ and an open region $\Re$ of the $n$-dimensional arithmetic number space $\left\{x^{i}\right\}$ exist such that $a_{\alpha \beta}\left(y^{\gamma}\right)$ are real functions of class $C^{2}$ and $y^{\alpha}\left(x^{i}\right)$ are real functions of class $C^{3}$ for $x \subset \Re$. Since the rank of $\left\|\partial y^{\alpha} / \partial x^{i}\right\|$ is $n$, (2) is non-singular for $x \subset \Re$ although it may be indefinite. Under these conditions the Gauss equations

$$
R_{h i j k}=e\left(\Omega_{h j} \Omega_{i k}-\Omega_{h k} \Omega_{i j}\right)+\bar{R}_{\alpha \beta \gamma \delta} y_{, h}^{\alpha} y_{, i}^{\beta} y_{, j}^{\gamma} y_{, k}^{\delta}
$$

are satisfied for $x \subset \Re$. In these equations $R_{h i j k}$ and $\bar{R}_{\alpha \beta \gamma \delta}$ are the

* Throughout this paper, Greek indices have the range $1,2, \cdots, n+1$ and Latin indices the range $1,2, \cdots, n$. An index which appears twice in an expression is to be summed over the appropriate range unless the index appears in parentheses.

$\dagger$ The comma denotes covariant differentiation with respect to the tensor $g_{i j}$. 
Riemann curvature tensors of $V_{n}$ and $V_{n+1}$ respectively, and $e$ is plus one or minus one, being defined by

$$
e=a_{\alpha \beta} \xi^{\alpha} \xi^{\beta}
$$

where the $\xi^{\alpha}$ are the components of the unit normal to $V_{n}$. The quantities $\Omega_{i j}$ are the coefficients of the second fundamental form of $V_{n}$

$$
\Omega_{i j}=a_{\alpha \beta} \xi^{\alpha} y_{, i j}^{\beta}+[\beta \gamma, \alpha]_{a} y_{, i}^{\beta} y_{, j}^{\gamma} \xi^{\alpha},
$$

where the brackets are the Christoffel symbols of the first kind formed with respect to $a_{\alpha \beta}$ and evaluated for $x \subset \Re$. It is clear that the functions $R_{h i j k}$ and $\bar{R}_{\alpha \beta \gamma \delta}$ are of class $C^{0}, \Omega_{i j}$ of class $C^{\prime}$, and $\xi^{\alpha}$ of class $C^{2}$ for $x \subset \Re$.

The directions of the lines of curvature of $V_{n}$ are given by the vectors* ${ }_{(p)} \lambda^{i}$ which satisfy

$$
\left(\Omega_{i j}-K_{p} g_{i j}\right)_{(p)} \lambda^{i}=0,
$$

where $K_{p}$ are the principal normal curvatures and are the roots of the determinant equation

$$
\left|\Omega_{i j}-K g_{i j}\right|=0 \text {. }
$$

If the elementary divisors of (8) are simple (as is always the case if (3) is definite), there is at least one orthogonal ennuple of unit vectors ${ }_{(p)} \lambda^{i}$ which satisfy $(7)$. When the elementary divisors are not simple, the tangents of some of the lines of curvature are null vectors. If (2) is indefinite, the principal normal curvatures need not be real even though $V_{n}$ and $V_{n+1}$ are both real Riemann spaces. The tangent of a line of curvature is real when and only when the corresponding principal normal curvature is real. $\dagger$

3. The theorem of Gauss for any Riemann space $V_{n+1}$. We assume that ${ }_{(1)} \lambda^{i}$ and ${ }_{(2)} \lambda^{i}$ are two unit vectors which are tangent to real lines of curvature at a point $P$ corresponding to $x \subset \Re$. If these directions and any $(n-2)$ real unit vectors orthogonal to both ${ }_{(1)} \lambda^{i}$ and (2) $\lambda^{i}$ are chosen as coordinate directions, it follows from the algebraic theory that at $P$.

$$
\begin{aligned}
g_{13} & =g_{14}=\cdots=g_{1 n}=0, & (1) & \lambda^{2}={ }_{(1)} \lambda^{3}=\cdots={ }_{(1)} \lambda^{n}=0 \\
g_{23} & =g_{24}=\cdots=g_{2 n}=0, & (2) & \lambda^{1}={ }_{(2)} \lambda^{3}=\cdots={ }_{(2)} \lambda^{n}=0, \\
\Omega_{1 i} & =K_{1} g_{1 i}, & \Omega_{2 i} & =K_{2} g_{2 i} .
\end{aligned}
$$

* Here $p$ denotes the vector and $i$ the component.

$\dagger$ T. J. I'A. Bromwich, Quadratic Forms and their Classification by Means of Invariant Factors, 1906, chaps. 3 and 4. Also cf. M. Bôcher, Introduction to Higher Algebra, 1929, p. 305, and Eisenhart, loc. cit., pp. 108-112. 
We denote by $k_{p q}$ the Riemannian curvature for the orientation determined by ${ }_{(p)} \lambda^{i}$ and ${ }_{(q)} \lambda^{i}$. By definition,

$$
k_{12}=\frac{R_{h i j k(1)} \lambda^{h}{ }_{(2)} \lambda^{i}{ }_{(1)} \lambda^{j}{ }_{(2)} \lambda^{k}}{\left(g_{h j} g_{i k}-g_{h k} g_{i j}\right)_{(1)} \lambda^{h}{ }_{(2)} \lambda^{i}{ }_{(1)} \lambda^{j}{ }_{(2)} \lambda^{k}} .
$$

From (4), (9), and (10) it follows that

$$
k_{12}=e K_{1} K_{2}+\frac{\bar{R}_{\alpha \beta \gamma \delta(1)} \xi_{(2)}^{\alpha} \xi_{(1)}^{\beta} \xi^{\gamma}{ }_{(2)} \xi^{\delta}}{g_{11} g_{22}-g_{12}^{2}},
$$

where ${ }_{(p)} \xi^{\alpha}={ }_{(p)} \lambda^{h} y_{, h}^{\alpha}$ is the component in the $y^{\prime}$ s of ${ }_{(p)} \lambda^{h}$. Since, from (3) and (9), $g_{11} g_{22}-g_{12}^{2}=\left(a_{\alpha \gamma} a_{\beta \delta}-a_{\alpha \delta} a_{\beta \gamma}\right)_{(1)} \xi_{(2)}^{\alpha} \xi_{(1)}^{\beta} \xi_{(2)}^{\gamma} \xi^{\delta}$, the last equation is equivalent to

$$
k_{12}-\bar{k}_{12}=e K_{1} K_{2},
$$

where $\bar{k}_{p q}$ is the Riemannian curvature of $V_{n+1}$ for the orientation determined by ${ }_{(p)} \xi^{\alpha}$ and ${ }_{(q)} \xi^{\alpha}$. Since all the quantities in (11) are invariants, this proves the theorem of Gauss for any $V_{n+1}$ and is valid at all points of $V_{n}$ for which $x \subset \Re$.

Let $V_{n}$ be a hypersurface of a Riemann space $V_{n+1}$. Then the difference of the Riemannian curvatures of the $V_{n}$ and the $V_{n+1}$ at a point for the orientation determined by the directions of two real lines of curvature of $V_{n}$ at the point, neither of which is tangent to a null vector, is numerically equal to the product of the corresponding normal curvatures; the sign is given by (5) and thus is determined by the character of the normal to $V_{n}$ in $V_{n+1}$.

4. The converse of Gauss' theorem for a flat $V_{n+1}$. If $V_{n+1}$ is a flat space, $\bar{k}_{p q} \equiv 0$ and

$$
k_{p q}=e K_{p} K_{q}
$$

for every hypersurface for which the elementary divisors of (8) are simple. This is the theorem of Gauss for a flat $V_{n+1}$. To prove the converse of this theorem, we first prove the lemma:

Given any Riemann space $V_{n+1}$, an arbitrary.point $P$ of this space, and a set of $n$ mutually orthogonal vectors ${ }_{(1)} \xi^{\alpha},{ }_{(2)} \xi^{\alpha}, \cdots,{ }_{(n)} \xi^{\alpha}$ at $P$; then there exists a hypersurface $V_{n}$ in $V_{n+1}$ such that $V_{n}$ contains the point $P$ and such that the lines of curvature of $V_{n}$ have the directions ${ }_{(1)} \xi^{\alpha},{ }_{(2)} \xi^{\alpha}, \cdots,{ }_{(n)} \xi^{\alpha}$ at $P$.

To prove this lemma we construct the $V_{n}$. Let the coordinates $y^{\alpha}$ of (1) be normal coordinates with the given point $P$ as center so 
that the given ennuple ${ }_{(p)} \xi^{\alpha}$ and the vector ${ }_{(n+1)} \xi^{\alpha}$ normal to ${ }_{(p)} \xi^{\alpha}$ at $P$ are the coordinate directions. Then at $P$,

$$
\begin{aligned}
& a_{\alpha \alpha}=e_{\alpha}, \quad a_{\alpha \beta}=0, \quad(\alpha) \xi^{\alpha}=1, \\
& (\beta) \xi^{\alpha}=0 ; \quad[\nu \mu, \gamma]_{a}=0, \quad \frac{\partial a_{\nu \mu}}{\partial y^{\gamma}}=0, \quad \alpha \neq \beta,
\end{aligned}
$$

where $e_{\alpha}=a_{\beta \gamma} \cdot(\alpha) \xi_{(\alpha)}^{\beta} \xi^{\gamma}$.

We define a hypersurface $S$ by the equations

$$
\begin{aligned}
y^{i} & =x^{i} \\
y^{n+1} & =\frac{1}{2} A_{i} x^{i^{2}},
\end{aligned}
$$

where the $A_{i}$ are non-zero constants. It is clear that ${ }_{(n+1)} \xi^{\alpha}$ is normal to $S$. From (3) and (13) it follows that at $P$

$$
g_{i i}=e_{i}, \quad g_{i j}=0,
$$$$
i \neq j \text {. }
$$

Upon differentiating (3) with respect to $x^{k}$, we have

$$
\frac{\partial g_{i j}}{\partial x^{k}}=\frac{\partial a_{\alpha \beta}}{\partial y^{\gamma}} y_{, k}^{\gamma} y_{, i}^{\alpha} y_{, j}^{\beta}+a_{\alpha \beta} \frac{\partial^{2} y^{\alpha}}{\partial x^{i} \partial x^{k}} y_{, j}^{\beta}+a_{\alpha \beta} y_{, i}^{\alpha} \frac{\partial^{2} y^{\beta}}{\partial x^{j} \partial x^{k}} .
$$

It follows from this equation, (13), and (14) that, for $x^{i}=0$,

$$
\frac{\partial g_{i j}}{\partial x^{k}}=0
$$

From (6), (13), (14), and (16), we have at $P$

$$
\Omega_{i i}=e_{n+1} A_{i}, \quad \Omega_{i j}=0,
$$$$
i \neq j \text {. }
$$

Hence from (15) and (17) it follows that $S$ is a $V_{n}$ which has the given vectors $(p) \xi^{\alpha}$ as the directions of its lines of curvature at the given point. This proves the lemma.

From this result, the converse of Gauss' theorem for a flat $V_{n+1}$, stated in the introduction, follows immediately. For if (12) holds for every hypersurface in $V_{n+1}$, the lemma shows that $\bar{k}_{p q}$ must be zero for an arbitrary orientation, hence $V_{n+1}$ is flat.

It is clear that a similar argument may be employed to show that if

$$
k_{p q}-k_{0}=e K_{p} K_{q}, \quad k_{0} \text { a constant, }
$$

for every hypersurface for which the elementary divisors of (8) are simple, $V_{n+1}$ has constant Riemannian curvature $k_{0}$ and conversely.

Princeton University AND

The Institute for Advanced Study 